\title{
Original investigation effects of Hydrogymnastics on igf-I and functional autonomy in elderly women
}

\begin{abstract}
Background \& aim: Decreases in the organic form and function inherent to the aging process tend to reduce the functional capacity and the insulin-like growth factor I (IGF-1) hormone levels in the elderly. This study aimed to evaluate the effects of hydrogymnastics on IGF-1 serum levels and functional autonomy (FA) in elderly women.
\end{abstract}

Methods: 28 elderly women were randomly divided into 2 groups: hydrogymnastics group (HG, $\mathrm{n}=14$; age: $66.55 \pm 3.77$ years; BMI: $27.03 \pm 5.44 \mathrm{~kg} / \mathrm{m}^{2}$ ) and control group $(\mathrm{CG}, \mathrm{n}=14$; age: $66.33 \pm 4.52$ years; BMI: $\left.29.82 \pm 4.96 \mathrm{~kg} / \mathrm{m}^{2}\right)$. The intervention with hydrogymnastics was performed 3 times per week during 12 weeks. Each session was composed of warming up, main part and cool-down, totaling 50 minutes of duration. The IGF-1 levels were analyzed by chemiluminescence. The GDLAM protocol of FA was applied - walking 10 $\mathrm{m}(\mathrm{W} 10 \mathrm{~m})$; rising from the sitting position (RSP); rising from a ventral decubitus position (RVDP); sitting in, rising from, and walking around a chair (SRWC); and putting on and taking off a t-shirt (PTTs) - and the overall autonomy index (AI) was calculated.

Results: The HG showed a significant increase in IGF-1 serum levels after the intervention when compared to CG. The execution times in the GDLAM protocol tests were significantly lower in the $\mathrm{HG}$, except in the SRWC, and the $\mathrm{HG}$ also presented a significant improvement in the AI post-intervention $(\mathrm{p}<0.05)$

Conclusion: The 12-week hydrogymnastics intervention contributed to an increase in IGF1 serum levels and to improve the performance in activities of daily living (ADL) in the elderly women.

Keywords: activities of daily living, aging, aquatic physical exercise, IGF-1, older women, water aerobics, GDLAM protocol
Volume I Issue 5 - 2017

\author{
Rodrigo Gomes de Souza Vale, ,2,4 Juliana \\ Brandão Pinto de Castro,' Rosana Dias \\ de Oliveira, ${ }^{2}$ Carlos Soares Pernambuco, ${ }^{2}$ \\ Flávio Boechat de Oliveira, ${ }^{1,2}$ Rafael da Silva \\ Mattos,' Dirceu Ribeiro Nogueira da Gama,' \\ Rodolfo de Alkmim Moreira Nunes,' Estélio \\ Henrique Martin Dantas ${ }^{3,4}$ \\ 'Department of Postgraduate Program in Exercise and Sport \\ Sciences, Rio de Janeiro State University, Brazil \\ ${ }^{2}$ Department of Laboratory of Exercise Physiology, Estácio de Sá \\ University, Brazil \\ ${ }^{3}$ Tiradentes University, Aracaju, Brazil \\ ${ }^{4}$ Laboratory of Biosciences and Human Motricity, Federal \\ University, Brazil
}

Correspondence: Rodrigo Gomes de Souza Vale, Department of Postgraduate Program in Exercise and Sport Sciences, Rio de Janeiro State University, Brazil, Email rodrigovale@globo.com

Received: May 30, 2017| Published: July 24, 2017

\section{Introduction}

Aging is a multidimensional phenomenon associated with the decline of organic form and function. The gradual and progressive physiological changes inherent to the aging process increase the prevalence of acute and chronic diseases. ${ }^{1,2}$ Among the alterations, there are deficits of balance, mobility, flexibility and muscular strength $^{3}$ postural control instabilities that can lead to changes in walking and posture ${ }^{4}$ endocrine abnormalities - such as the reduction of serum levels of anabolic hormones, as the insulin-like growth factor I (IGF-1 $)^{5,6}$ and changes in body composition, including decreases in muscle and bone masses. ${ }^{7}$

These morpho functional declines tend to cause loss of independence in the elderly and, consequently, the decrease in the functional capacity to perform the activities of daily living (ADL). ${ }^{2}$ It tends to increase the risk of falls, which can cause serious health consequences, either due to the fall itself, such as fractures, or fall aggravations. ${ }^{8,9}$

Aging-related IGF-1 reduction may be associated with loss of lean mass, decreased protein synthesis, and increased adipose mass in elderly individuals. ${ }^{10,11}$ The IGF-1 provides information about bone mineral density (BMD) ${ }^{12}$ and performs an anabolic effect on the osteoblasts, increasing the cellular proliferation and estimating the synthesis of the bone mineral matrix. ${ }^{13}$

The regular practice of physical exercise and activity can improve health, functional autonomy (FA) and independence in this population. ${ }^{14}$ In this sense, physical activity has been an efficient strategy to improve FA and IGF-1 hormone levels in the elderly. ${ }^{15}$

The numerous restrictions to which the elderly are susceptible should be considered in the prescription and in the choice of exercises. Hydrogymnastics is an activity that is highly sought by the elderly. ${ }^{16}$ The reasons involve social interaction, improvement of the quality of life and the pleasure of performing physical activities in the aquatic environment. ${ }^{17}$ Some advantages of hydrogymnastics compared to land-based exercises are the possibility of increased load with lower risk of injury and greater comfort due to the appropriate water temperature ${ }^{18} \mathrm{~A}$ randomized controlled tria ${ }^{19}{ }^{19}$ investigated the effects of 10 months of aquatic exercises on BMD, and found no significant improvement in BMD in postmenopausal women with osteoporosis. Similar results were found in a study ${ }^{20}$ that evaluated the effects of eight-month aquatic exercise program on BMD in older women with low BMD. The training did not improve lumbar and total femur BMD. However, another randomized controlled trial ${ }^{21}$ evaluated the 24-week effects of a high intensity aquatic exercise program on BMD of postmenopausal women. This intervention was efficient in attenuating bone resorption raise and enhancing bone formation, which prevented the participants from reducing the femoral trochanter BMD.

Improved FA and hormonal levels, induced by a physical activity program, seems to be important for maintaining good performance in ADL and decreasing the chances of physical dependence in elderly women. Therefore, the aim of the present study was to evaluate the effects of hydrogymnastics on IGF-1 serum levels and FA in elderly women. 


\section{Materials and methods}

\section{Participants}

This is an experimental study with simple random sampling. The universe of the present study was constituted of 95 elderly subjects aged 60 years or older residing in Araruama, RJ - Brazil. All of them lived in their homes and had FA to perform ADL independently, without help from other people. In order to capture the sampling group, lectures were given at the Municipal Theater in the city of Araruama. No male subjects attended the call at the Municipal Theater. Therefore, the sample of the present study consisted of female subjects between the ages of 60 and 70 years who resided in this city. The changes inherent to the aging process, such as changes to hormone systems that affect the performance of ADL, occur earlier in women than in men, with the functional decrease initiating around menopause. ${ }^{1}$

The sample was randomly selected from women who had volunteered to participate in the exercise program. The inclusion criteria were women aged 60 years or more who were: a) physically able to participate in the experimental treatment; b) independent in the performance of ADL; c) not practicing any systematized physical activity for at least six months; d) not using additional bone active medications or hormone replacement therapy; and e) considered able, based on a medical evaluation, to engage in physical activity.

The study adopted as exclusion criteria: a) any type of acute or chronic condition that could compromise the performance or become an incapacitating factor to participate of an aquatic program, such as heart disease, diabetes, arterial hypertension and non-controlled asthma; b) any musculoskeletal conditions that could represent an intervening factor in the practice of hydrogymnastics (osteoarthritis, recent fracture, tendinitis and use of prostheses); and c) current neurological problems or use of medications that could cause attention disturbance. The sample size (n) was calculated through a pilot study following the recommendations of Barreto \& Ribeiro. ${ }^{22}$ Considering the inclusion and exclusion criteria and the calculation of the sample size, the sample of this study consisted of 28 elderly women who were randomly divided by simple draw into a group that participated in a hydrogymnastics program $(\mathrm{HG}, \mathrm{n}=14)$, and a control group $(\mathrm{CG}$, $\mathrm{n}=14$ ) who engaged in no formal physical activity program. The $\mathrm{CG}$ was instructed to maintain their normal daily tasks throughout the study period. The elderly women in the CG were invited to participate in the hydrogymnastics program after the intervention period of the study.

The participants who agreed to participate in the study signed an informed consent form in accordance with the guidelines regarding human research delineated in the Resolution 466/2012 of the National Health Council and the Declaration of Helsinki. The Estácio de Sá University Research Ethics Committee, under the number 1.617.605, approved this research.

\section{Evaluation of anthropometric measurements}

To measure the body mass and the height of the sample, we used a mechanical scale with stadiometer (Filizola, Brazil), with a scale precision of $100 \mathrm{~g}$. Additionally, we calculated the body mass index (BMI) as the ratio between the body mass and the square of the height $\left(\mathrm{kg} / \mathrm{m}^{2}\right)$. All procedures were performed by a single evaluator and strictly followed the standardization of the International Society for Advancement in Kinanthropometry (ISAK). ${ }^{23}$

\section{Functional autonomy}

FA was analyzed through the GDLAM protocol, ${ }^{1,24,25}$ consisting of five tests: 1) walking $\left.10 \mathrm{~m}(\mathrm{~W} 10 \mathrm{~m})^{26} 2\right)$ rising from the sitting position (RSP) ${ }^{27} 3$ ) rising from a ventral decubitus position (RVDP). ${ }^{28}$ 4) sitting in, rising from, and walking around a chair (SRWC) ${ }^{29}$ and 5) putting on and taking off a t-shirt (PTTs).$^{30}$ This protocol was created and validated by the Group of Latin-American Development for Maturity (GDLAM), hence called the GDLAM protocol. ${ }^{1,24}$ Each task was performed twice with at least five minutes of interval between the tasks. The shortest time was recorded using a chronometer (Casio, Brazil). The GDLAM autonomy index (AI) was calculated ${ }^{25}$ by a normalization process between the five autonomy tests to estimate a value in scores. This calculation was made using the formula proposed below, where W10 m, RSP, RVDP, PTTs, and SRWC correspond to the time measured in seconds, and AI is the GDLAM index in scores. A lower AI score designates greater levels of FA and vice versa.

$$
\mathrm{AI}=\frac{[(\mathrm{W} 10 \mathrm{~m}+\mathrm{RSP}+\mathrm{RVDP}+\mathrm{PTTs}) \times 2]+\mathrm{SRWC}}{4}
$$

\section{IGF-I assessment}

Basal serum IGF-1 levels were analyzed in a clinical analysis laboratory using the Immulite - DPC Med Lab Chemiluminescence method. Blood samples were taken at rest in the morning, where complete fasting was not required, only milk and egg products were avoided. Blood samples, at least $5 \mathrm{ml}$, were collected in a dry tube and the serum were separated and sent for processing. The collection was done in venipuncture. The collected blood was immediately centrifuged at $3,500 \mathrm{rpm}$ for 10 minutes to obtain at least $1 \mathrm{ml}$ of serum. All tests were confirmed. The serum of each sample was placed on a rack with proper identification. The analysis was done by the chemiluminescence method during a period of 3 minutes. The results were sent to a central computer by interfacing. The reference values used $(69-200 \mathrm{ng} / \mathrm{ml})$ for analyses were obtained according to the recommendations of Huayllas et al. ${ }^{31}$

\section{Training program}

The HG performed a water aerobics exercise program in a heated pool $\left(27\right.$ to $\left.29^{\circ} \mathrm{C}\right)$ with a depth of 1.00 to $1.40 \mathrm{~m}$, with subjects positioned in a way that the water slide remained level with the axillary line of the body. The hydrogymnastics program lasted 12 weeks and consisted of 3 weekly sessions on alternate days. Each session was composed of warm up (10minutes), main part (35minutes) and cooldown (5minutes), totaling 50minutes of duration.

The warm up consisted of lateral steps, walking in different directions with alternation of several movements of the arms and legs. The main part included 5 phases ( 7 minutes each) with the following exercises: 1) ski movement (drag), stationary running with variations of hip flexion movements and knee flexion without and with trunk rotation and elbows flexed at $90^{\circ}$ (elbow toward the knee); 2) stationary running with elbow flexion and alternating movements with legs extended and flexed, leaps forward with alternation of extended legs and slight knee and plantar flexion; 3) varied jumps with hip hyperextension, with plantar flexion, and with isometric muscular contraction of gluteus and quadriceps; 4) stationary running while alternating elbow flexion and extension, and ski movement with horizontal flexion and extension of the shoulder (both exercises with the use of dumbbells); and 5) alternation of front, side, and back kicks 
with loose arms. The cool-down consisted of walking and muscle release exercises.

Movement patterns were used allowing sufficient time for transitions and readjustment of body alignment. Exercises in the three movement plans were prioritized to help maintain or improve the amplitude of the joints. Accessories, as dumbbells, were used to increase the effort level of the training sessions. These devices were introduced at a specific time for all participants.

The exercises were performed in three sets of 15 to 20 repetitions or per time with a load representing a comfortable level of effort intensity. Training load was controlled by maintaining the perceived effort (on the Borg CR10 scale) ${ }^{32}$ between levels 2 and 3 (mild to moderate intensity) in the initial 4 weeks and between 3 and 5 (moderate intensity) in the remaining weeks until the end of the intervention period.

\section{Statistical analysis}

Descriptive statistics presented the mean, standard deviation, minimum and maximum values for subject characteristics and dependent variables. The Shapiro-Wilk and Levene tests were used to verify the normality and variance homogeneity of the data, respectively. The ANOVA with repeated measures was used to compare changes within and between treatment groups (HG vs. CG) over time (prevs. post-intervention). The Turkey's post hoc procedure was used to identify the possible differences of these comparisons. The study adopted $\mathrm{p}<0.05$ as the significance level. The power of the experiment and the beta error ( $\beta$-error) were calculated to analyze the sample size.
Statistical analyses of all data were performed using the IBM SPSS Statistics 20 for Windows.

\section{Results}

The power of the experiment achieved in this study was $82 \%$ to the FA, presenting a calculated beta ( $\beta$ ) of 0.18 , and $90 \%$ to the IGF-1, presenting a calculated beta $(\beta)$ of 0.10 . Thus, the research can sustain the results with the sample size used. The descriptive characteristics of the sample are presented in Table 1. The p-values (Shapiro-Wilk test) indicate that the groups ( $\mathrm{HG}$ and $\mathrm{CG}$ ) were similar in the beginning of the present study.

Table I Characteristics of the sample

\begin{tabular}{lllllll}
\hline Group & Variables & Mean & SD & Min & Max & $\begin{array}{l}\text { p-value } \\
\text { (SW) }\end{array}$ \\
\hline HG & Age (years) & 66.55 & 3.77 & 60 & 69 & 0.822 \\
& BMl (kg/m2) & 27.03 & 5.44 & 21.87 & 32.63 & 0.483 \\
CG & Age (years) & 66.33 & 4.52 & 61 & 70 & 0.192 \\
& BMl (kg/m2) & 29.82 & 4.96 & 25.76 & 34.21 & 0.482 \\
\hline
\end{tabular}

SD, Standard Deviation; Min, Minimum; Max, Maximum; BMI, Body Mass Index; SW, Shapiro-Wilk

Table 2 presents the intragroup results of FA through the GDLAM protocol tests. In the HG, all GDLAM protocol tests and AI presented a significant reduction in the execution times and scores, respectively. On the other hand, CG showed no changes.

Table 2 Functional autonomy analysis in hydrogymnastics group (HG) and control group (CG)

\begin{tabular}{llllllll}
\hline Group & Variables & Mean (pre) & SD & Mean (post) & SD & $\Delta$ & P-value \\
\hline HG & WIOm & 7.2 & 0.94 & 6.03 & 1.11 & -1.17 & $<0.001$ \\
& RSP & 11.65 & 2.4 & 10.04 & 0.9 & -1.61 & 0.042 \\
& RVDP & 3.67 & 0.79 & 2.84 & 0.6 & -0.83 & 0.004 \\
& PTTs & 11.02 & 2.26 & 9.8 & 2.06 & -1.22 & 0.013 \\
& SRWC & 46.11 & 4.26 & 42.33 & 4.42 & -3.78 & 0.003 \\
CG & AI & 28.3 & 2.95 & 24.93 & 2.88 & -3.37 & $<0.001$ \\
& WIOm & 7.44 & 0.83 & 7.36 & 1.11 & -0.08 & 0.789 \\
& RSP & 11.09 & 1.78 & 11.95 & 2.01 & 0.87 & 0.174 \\
& RVDP & 3.74 & 0.91 & 4.07 & 1.01 & 0.33 & 0.321 \\
& PTTs & 10.83 & 1.78 & 12.33 & 2.09 & 1.5 & 0.076 \\
& SRWC & 46.07 & 5.16 & 45.49 & 6.34 & -0.58 & 0.747 \\
& Al & 28.07 & 2.8 & 29.23 & 3.5 & 1.16 & 0.325 \\
\hline
\end{tabular}

W10m: walking $10 \mathrm{~m}$; RSP: rising from the sitting position; RVDP: rising from a ventral decubitus (prone) position; PTTs: putting on and taking off a t-shirt; SRWC: sitting in, rising from, and walking around a chair; AI: GDLAM autonomy index; SD: standard deviation; $\Delta$ : absolute difference. Values in seconds. AI: values in scores.

Table 3 shows the results of the intergroup comparisons in the variable FA through the percentage differences. As indicated by this

table, the HG obtained a significant reduction in the execution times of the GDLAM protocol, except in the SRWC, and in the scores of AI when compared to $\mathrm{CG}$.

W10m: walking $10 \mathrm{~m}$; RSP: rising from the sitting position; RVDP: rising from a ventral decubitus (prone) position; PTTs: putting on and taking off a t-shirt; SRWC: sitting in, rising from, and walking around a chair; AI: GDLAM autonomy index; $\Delta \%$ : percentage difference. 
Table 3 Percent difference analysis in the HG and the CG

\begin{tabular}{llll} 
Variables & $\Delta \%$ HG & $\Delta \%$ CG & p-value \\
\hline WIOm & -16.27 & -1.12 & 0.044 \\
RSP & -13.85 & 7.82 & 0.021 \\
RVDP & -22.69 & 8.94 & 0.016 \\
PTTs & -11.08 & 13.87 & 0.039 \\
SRWC & -8.2 & -1.26 & 0.056 \\
Al & -11.89 & 4.15 & 0.026
\end{tabular}

Figure 1 shows the intra and intergroup comparisons of the GDLAM autonomy index (AI). The AI was reduced to a significantly greater extent in the HG group when compared to the CG group which actually increased slightly.

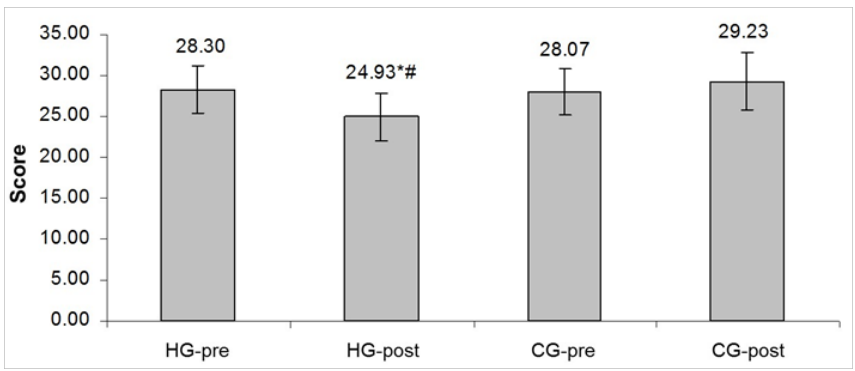

Figure I Comparative analysis of GDLAM autonomy index (Al).

$*_{\mathrm{p}}<0.05$; HG-pre vs HG-post.

$\# p<0.05 ;$ HG-post vs CG-post.

Figure 2 shows the intra- and inter-group comparisons of IGF-1 levels of both groups. The IGF-1 levels increased to a significantly greater extent in $\mathrm{HG}$ after completion of the hydrogymnastics program. Changes within the CG were not statistically significant.

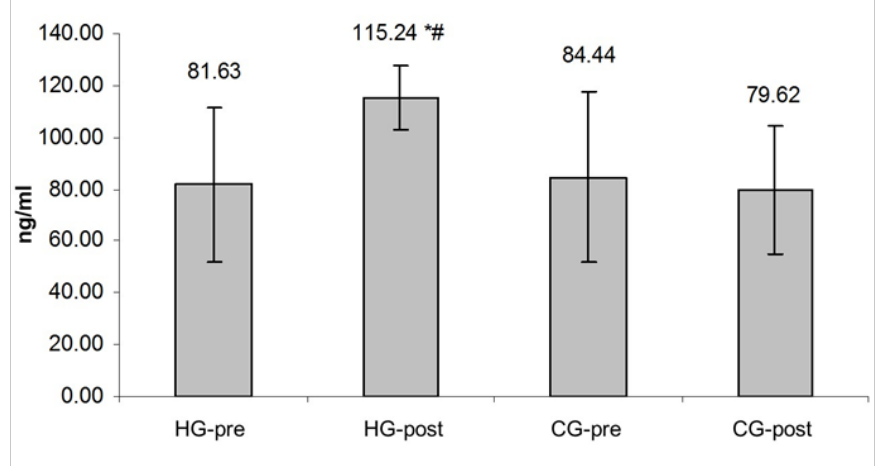

Figure 2 Comparative analysis of IGF-I levels.

$*_{p}<0,05$; HG-pre vs HG-post.

\#p $<0,05 ;$ HG-post vs CG-post.

\section{Discussion}

The results of the present study show a significant difference for the IGF-1 serum variable in the HG group. These results are consistent with the study of Vale et al. ${ }^{33}$ who observed a positive correlation between IGF-1 and FA for ADL. The elderly who participated in that study were more active, which explains the higher IGF-1 values.

In a study by Maïmoun et al. ${ }^{34}$ involving a group of elderly people who performed strenuous physical activity, a change in bone metabolism was observed using distinct markers (blood calcium ions). Serum changes also occurred in the group analyzed in this study, demonstrating that physical exercise promoted beneficial changes in bone metabolism. Because bone mass typically decreases with age, older adults typically have a greater risk of sustaining a fall-related fracture. ${ }^{35}$

Accordingly, activities such as dance and strength training have been shown to promote significant changes of IGF-1 levels in the elderly. Pires \& Dantas ${ }^{36}$ observed similar results, corroborating the findings of the present study, and demonstrating that several modalities of physical exercise can promote health benefits and functional mobility among this age group. The results found in the current study showed significant improvements in the FA variables related to ADL in the HG. The variables W10m, RSP, RVDP, and PTTs showed significantly better changes when compared with the CG. Passos et al. ${ }^{37}$ also found significant improvements in the performance of ADL among elderly women who practiced hydrogymnastics. That intervention program had the same frequency and duration used in the present study. The initial AI of the present study described the group as weak, similar to the group studied by Alencar et al. ${ }^{38}$ These authors ${ }^{38}$ with a sample of 15 elderly women, observed that the indicators of autonomy to perform daily life activities by the GDLAM protocol achieved unsatisfactory rates, reinforcing the premise that aging associated with a sedentary lifestyle causes reduced functional capacity. Initially, the HG of the present study was sedentary and, after the intervention, the same group was qualified as good. This demonstrates that the activity developed promoted beneficial changes in FA.

The findings of the current study are also corroborated by the results found by Silva et al. ${ }^{39}$ who studied a similar age group and also reported significant improvements in FA following a strength training program. Although the exercise intervention is different, the present investigation reached, through a hydrogymnastics program, satisfactory levels of FA in the HG.

Despite its positive findings, the current study did have some limitations. The sample size was small and included only female participants, which limits the external validity of these results. The serum IGF-1 levels can be altered by a variety of, many of which could not be controlled such as the type of diet, lifestyle, stress, sleep, alcohol intake. ${ }^{40}$ On the other hand, the current study established the composition of a homogeneous group in order to maintain an ecological validity. In addition, the methodologic procedure adopted in the present study has a clear practical applicability in gym, conditioning and rehabilitation centers.

Based on the results found in the present study, it is possible to derive benefits in bone metabolism and FA via participation in a hydrogymnastics activity program. Aquatic activities promoted serum changes that indicate an anabolic effect on muscle and bone. The FA oriented to the performance of ADL of the experimental group studied was benefited by the practice of physical exercise, which tends to facilitate the life of the elderly, making them more autonomous.

Therefore, we recommended the implementation of hydrogymnastics in social projects that involve physical and 
recreational activities for the elderly due to the positive results observed in the current study. These benefits can change the lifestyle of these people and reduce the possibility of clinical institutionalization of the elderly. Further studies are recommended to evaluate the quality of life of the elderly and to analyze the bone mineral density and the behavior of other hormones, such as estradiol, T3 and T4, which tend to decrease with age.

\section{Acknowledgements}

None.

\section{Conflict of interest}

Author declares there is no conflict of interest in publishing the article.

\section{References}

1. Dantas EHM, Figueira HA, Emygdio RF, et al. Functional autonomy GDLAM protocol classification pattern in elderly women. Indian Journal of Applied Research. 2014;4(7):262-266.

2. Daniel FNR, Vale RGS, Giani TS, et al. Correlation between static balance and functional autonomy in elderly women. Arch Gerontol Geriatr. 2011;52(1):111-114.

3. Locks RR, Costa TC, Koppe S, et al. Effects of strength and flexibility training on functional performance of healthy older people. Braz J Phys Ther. 2012;16(3):184-190.

4. Daniel F, Vale R, Giani T, et al. Effects of a physical activity program on static balance and functional autonomy in elderly women. Maced $J$ Med Sci. 2010;3(1):21-26.

5. Fornelli G, Isaia GC, D Amelio P. Ageing, muscle and bone. Journal of Gerontology and Geriatrics. 2016;64(3):75-80.

6. Moran S, Chen Y, Ruthie A, et al. Alterations in IGF-I affect elderly: role of physical activity. Eur Rev Aging Phys Act. 2007;4(2):77-84.

7. Frontera WR, Hughes VA, Fielding RA, et al. Roubenoff R. Aging of skeletal muscle: a 12-yr longitudinal study. J Appl Physiol. 2000;88(4):1321-1326.

8. Boongird C, Keesukphan P, Phiphadthakusolkul S, et al. Effects of a simple home-based exercise program on fall prevention in older adults: A 12-month primary care setting, randomized controlled trial. Geriatr Gerontol Int. 2017.

9. Aslan UB, Cavlak U, Yagci N, et al. Balance performance, aging and falling: a comparative study based on a Turkish sample. Arch Gerontol Geriatr. 2008;46(3):283-292.

10. Oliveira LSC, Côrtes GG, Vale RGS, et al. Níveis séricos de IGF-1 em gerontes. Fit Perf J. 2003;2(5):289-291.

11. Arvat E, Broglio F, Ghigo E. Insulin-like growth factor-I: implications in aging. Drugs Aging. 2000;16(1):29-40.

12. Snow CM, Rosen CJ, Robinson TL. Serum IGF-I is higher in gymnasts than runners and predicts bone and lean mass. Med Sci Sports Exerc. 2000;32(11):1902-1907.

13. Olney RC. Regulation of bone mass by growth hormone. Med Pediatr Oncol. 2003;41(3):228-234.

14. Chodzko ZWJ, Proctor DN, Fiatarone SMA, et al. American College of Sports Medicine Position stand. Exercise and physical activity for older adults. Med Sci Sports Exerc. 2009;41(7):1510-1530.

15. Vale RGS, Oliveira RD, Pernambuco CS, et al. Effects of muscle strength and aerobic training on basal serum levels of IGF-1 and cortisol in elderly women. Arch Gerontol Geriatr. 2009;49(3):343-347.
16. Lima VP, Nunes RAM, Castro JBP, et al. Hemodynamic variations in elderly before and after exercises in hydrogymnastics. $J$ Phys Ed. 2017;86(1):18-24.

17. Mazo GZ, Cardoso FL, Aguiar D. Water gymnastics program for elderly: motivation, self-steam and self-image. Brazilian J Kinanthropmetry Human Perfor. 2006;8(2):67-72.

18. Alves RV, Mota J, Costa MC, et al. Physical fitness and elderly health effects of hydrogymnastics. Rev Bras Med Esporte. 2004;10(1):38-43.

19. Murtezani A, Nevzati A, Ibraimi Z, et al. The effect of land versus aquatic exercise program on bone mineral density and physical function in postmenopausal women with osteoporosis: a randomized controlled trial. Ortop Traumatol Rehabil. 2014;16(3):319-325.

20. Pernambuco CS, Vale RG, Di Masi F, et al, Functional autonomy, bone mineral density (BMD) and serum osteocalcin levels in older female participants of an aquatic exercise program (AAG). Arch Gerontol Geriatr. 2013;56(3):466-471.

21. Moreira LD, Fronza FC, Santos RN, et al. The benefits of a high-intensity aquatic exercise program (HydrOS) for bone metabolism and bone mass of postmenopausal women. J Bone Miner Metab. 2014;32(4):411-419.

22. Barreto ACLG, Ribeiro LG. Determinação do tamanho amostral. Fit Perf J. 2004;3(3):124.

23. Marfell JM, Olds T, Stewart A, et al. International standards for anthropometric assessment. Potchefstroom, South Africa; 2006.

24. Dantas EHM, Vale RGS. Protocolo GDLAM de avaliação de autonomia funcional. Fit Perf J. 2004;3(3):169-180.

25. Vale RGS. Avaliação da autonomia funcional do idoso. Fit Perf J. 2005;4(1):4.

26. Sipilä S, Multanen J, Kallinen M, et al. Effects os strength and endurance training on isometric muscle strength and walking speed in elderly women. Acta Physiol Scand. 1996;156(4):457-464.

27. Guralnik JM, Simonsick EM, Ferrucci L, et al. A short physical performance battery assessing lower extremity function: association with self-reported disability and prediction of mortality and nursing home admission. J Gerontol. 1994;49(2):85-94.

28. Alexander NB, Ulbric HJ, Raheja A, et al. Rising from the floor in older adults. J Am Geriatr Soc. 1997;45(5):564-569.

29. Andreotti RA, Okuma SS. Validação de uma bateria de testes de atividades da vida diária para idosos fisicamente independentes. Rev Paul Educ Fís. 1999;13(1):46-66.

30. Vale RGS, Pernambuco CS, Novaes JS, et al. Functional autonomy test: to dress and undress a sleeveless shirt (DUSS). Rev Bras Ciênc Mov. 2006;14(3):71-78.

31. Huayllas MKP, Carvalhaes NN, Ramos LR, et al. Níveis séricos de hormônio de crescimento, fator de crescimento símile à insulina e sulfato de deidroepiandrosterona em idosos residentes na comunidade. Correlação com parâmetros clínicos. Arq Bras Endocrinol Metab. 2001;45(2):157-166.

32. Borg GA. Psychophysical bases of perceived exertion. Med Sci Sports Exerc. 1982;14(5):377-381.

33. Vale RGS, Oliveira RD, Pernambuco CS, et al. Correlation between basal serum IGF-1 levels and functional autonomy in elderly women. Rev Int Cienc Deporte. 2009;5(14):11-18.

34. Maïmoun L, Simar D, Malatesta D, et al. Response of bone metabolism related hormones to a single session of strenuous exercise in active elderly subjects. Br J Sports Med. 2005;39(8):497-502.

35. Colaianni G, Colucci S, Cinti S, et al. The myokine Irisin recapitulates the effect of physical activity on bone and muscle tissues. Journal of Gerontology and Geriatrics. 2016;64(3):92-96. 
36. Pires T, Dantas EHM. Comparison of the basal serum levels of IGF-1 in elderly women practitioners of weight training, dance and meditation programs at Casa do Idoso in the city of Belém-PA, Brazil. Revista de Desporto e Actividade Física. 2009;2(1):1-6.

37. Passos BMA, Souza LHR, Silva FM, et al. Contributions of hidrogymnastic to activities of daily living and flexibility in older women. J Phys Ed. 2008;19(1):71-76.

38. Alencar NA, Bezerra JCP, Dantas EHM. Avaliação dos níveis de atividade física, autonomia funcional e qualidade de vida de idosas integrantes da estratégia saúde da família. Fit Perf J. 2009;8(5):315-321.
39. Silva JGBF, Cader AS, Dopico X, et al. Fortalecimiento muscular, nível de fuerza muscular y autonomía funcional em uma población de mujeres mayores. Rev Esp Geriatr Gerontol. 2009;44:256-261.

40. Bastos CLB, Miranda H, Vale RGS, et al. Winchester JB. Chronic effect of static stretching on strength performance and basal serum IGF-1 levels. J Strength Cond Res. 2013;27(9):2465-2472. 